\title{
Immediate Euglycemic Diabetic Ketoacidosis After Gastric Bypass in a Patient with Type II Diabetes
}

\author{
Yu-Ting Lo ${ }^{1} \cdot$ Kai-Hua Chen ${ }^{1} \cdot$ Po-Chih Chang ${ }^{2,3,4,5}$ (D) \\ Received: 10 January 2021 / Revised: 23 January 2021 / Accepted: 25 January 2021 / Published online: 10 February 2021 \\ (C) The Author(s) 2021
}

Keywords Bariatric surgery · Diabetic ketoacidosis · Euglycemic diabetic ketoacidosis · Type II diabetes mellitus

Bariatric surgery has been proven to be an effective solution for morbid obesity with type 2 diabetes mellitus (T2DM), and such patients usually receive oral hypoglycemia agents or insulin for blood sugar control prior to surgery [1]. Though the occurrence of euglycemic diabetic ketoacidosis (DKA) is not clinically infrequent especially for those on treatment with sodium-glucose cotransporter 2 (SGLT2) inhibitor, reports of DKA after bariatric surgery are limited and most of the available reports discussed type I diabetes [1-3].

Herein, we report a T2DM patient (without SGLT2 inhibitor use) who developed euglycemic DKA soon after Rouxen-Y gastric bypass (RYGB). Moreover, the postoperative course was complicated with an attack of chronic gouty arthritis and acute exacerbation of chronic kidney disease, but eventually the patient made a full recovery.

A 52-year-old, morbidly obese male patient (body weight $110.3 \mathrm{~kg}$; body mass index $37.7 \mathrm{~kg} / \mathrm{m}^{2}$ ), with newly diagnosed T2DM (glycated hemoglobin 9.5\%; homeostatic model assessment of insulin resistance 8.2) and poly-morbidities

Po-Chih Chang

dr.changpochih@hotmail.com

1 Department of Surgery, Kaohsiung Medical University Hospital/ Kaohsiung Medical University, Kaohsiung City, Taiwan

2 Division of Thoracic Surgery, Department of Surgery, Kaohsiung Medical University Hospital/Kaohsiung Medical University, Kaohsiung City, Taiwan

3 Weight Management Center, Kaohsiung Medical University Hospital/Kaohsiung Medical University, No. 100, Tzyou 1st Road, Kaohsiung City 80756, Taiwan

$4 \mathrm{Ph}$. D. Program in Biomedical Engineering, College of Medicine, Kaohsiung Medical University, Kaohsiung City, Taiwan

5 Department of Sports Medicine, College of Medicine, Kaohsiung Medical University, Kaohsiung City, Taiwan (hypertension, dyslipidemia, hyperuricemia with left ankle tophi, chronic kidney disease, and non-alcoholic fatty liver disease), was referred to our hospital for weight loss surgery. For blood sugar control, only metformin was prescribed $(500 \mathrm{mg}$ thrice a day) instead of a SGLT2 inhibitor. The patient had also taken diclofenac occasionally to relieve pain from chronic gouty arthritis over the bilateral feet. Laparoscopic RYGB was uneventfully performed on the 2nd day after admission with an estimated blood loss of $30 \mathrm{~mL}$. The operation time was $210 \mathrm{~min}$, and the intra-abdominal pressure of the pneumoperitoneum was up to $15-17 \mathrm{mmHg}$ during the surgery.

On postoperative day 1 , the patient started sipping water without obvious discomfort, and regular insulin was continuously infused at a rate of $1.25 \mathrm{U} / \mathrm{h}$ (blood sugar level was between 192 and $205 \mathrm{mg} / \mathrm{dL}$ ) (Fig. 1). However, a spiking fever $\left(39.3^{\circ} \mathrm{C}\right)$ occurred in the evening with a significant decrease in urine amount (from 3010 to $480 \mathrm{~mL} /$ day). Meanwhile, the patient also presented with tachypnea (26 breaths per minute) and tachycardia (123 beats per minute). No peritoneal sign or chest tightness was reported. The laboratory studies showed leukocytosis, elevated level of procalcitonin $(3.4 \mathrm{ng} / \mathrm{mL})$, and deteriorated renal function (creatinine $3.99 \mathrm{mg} / \mathrm{L}$ ) (Table 1). Moreover, the blood gas analysis demonstrated metabolic acidosis with respiratory compensation (Table 1). The blood sugar still remained at a marginally higher level $(219 \mathrm{mg} / \mathrm{dL})$. Upon arriving at the intensive care unit, hypotension developed $(72 / 49 \mathrm{mmHg})$ and fluid resuscitation, vasopressor, and empirical antibiotics (imipenem + cilastatin) were administered for possible occult intra-abdominal infection.

On the following day (postoperative day 2), blood ketone levels were examined for fluctuating blood sugar levels, which disclosed a positive result $(6.1 \mathrm{mmol} / \mathrm{L})$ in favor of DKA. Regular insulin pump was titrated up to $2 \mathrm{U} / \mathrm{h}$ accordingly. Thereafter, the fever subsided and urine output increased gradually with concomitant recovery of blood 


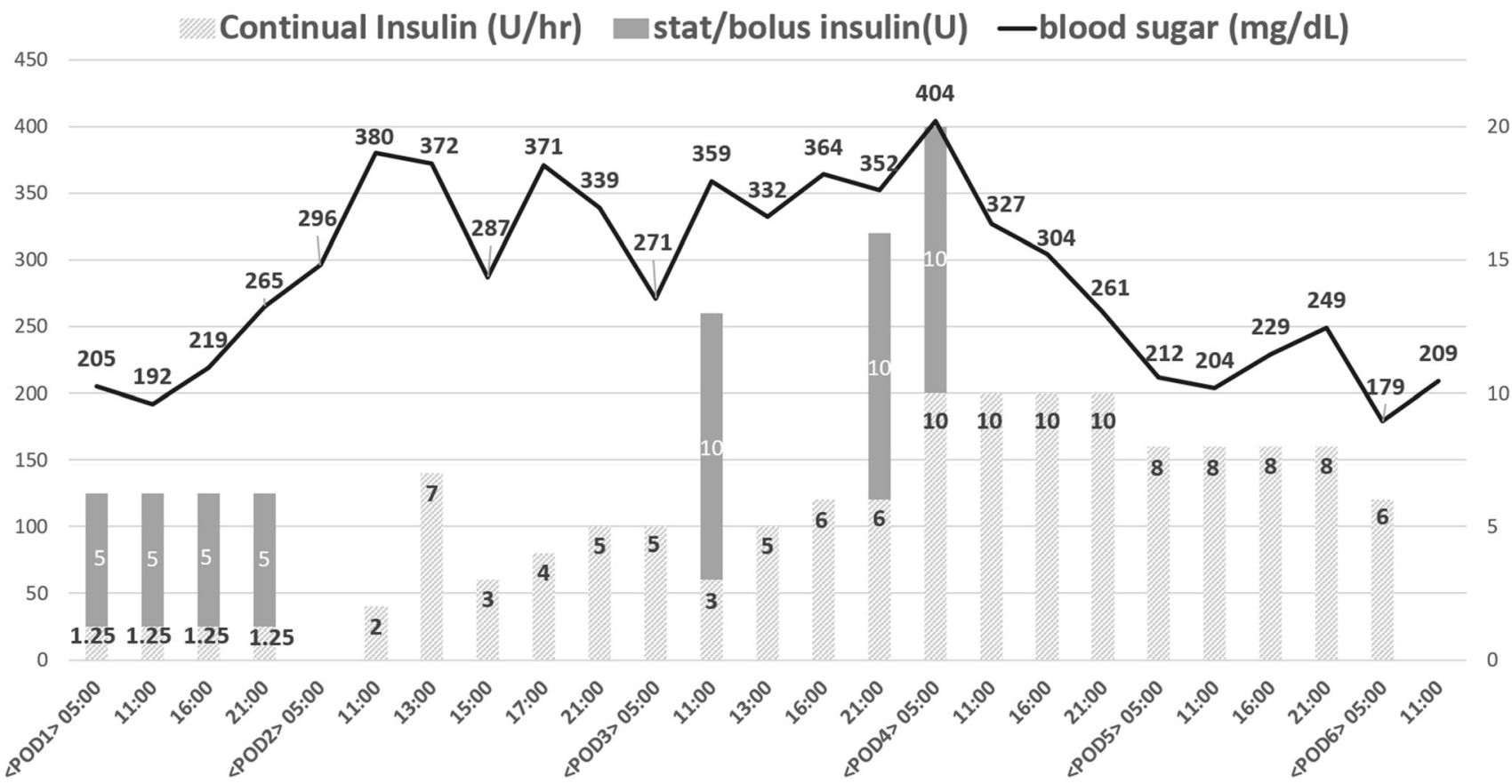

Fig. 1 Timeline of blood sugar level and regular insulin dose within the early postoperative stage. We infused regular insulin with a pump from postoperative day 2 to 6

pressure. Despite the occurrence of another episode of fever $\left(38.3^{\circ} \mathrm{C}\right)$ with significantly elevated procalcitonin $(37.04 \mathrm{ng} /$ $\mathrm{mL})$ and C-reactive protein $(291.41 \mathrm{mg} / \mathrm{L})$ on postoperative day 5 , in favor of a gout attack (blood uric acid $7.2 \mathrm{mg} / \mathrm{dL}$ ), a non-enhanced abdominal computed tomography (CT) was still arranged in order to detect possible occult intraabdominal infection or anastomotic leak. CT demonstrated neither fluid accumulation nor pneumoperitoneum.
Therefore, the patient was immediately started with clear liquid diet and shifted to a full liquid diet on postoperative day 8 with the basal-bolus regular insulin regimen.

On postoperative day 10 , we discontinued antibiotic use after the C-reactive protein and procalcitonin levels returned to the normal. His blood sugar remained stable after titration of insulin and oral hypoglycemic agent according to the endocrinologist's suggestion. The patient was discharged on

Table 1 Laboratory studies from postoperative day 1 to day 5. $p H$ potential of hydrogen, $p C O 2$ partial pressure of carbon dioxide, $B U N$ blood urea nitrogen, $e G F R$ estimated glomerular filtration rate

\begin{tabular}{|c|c|c|c|c|c|c|c|}
\hline & POD1 & & POD2 & & POD3 & POD4 & POD5 \\
\hline Serum glucose level (mg/dL) & 205 & 219 & 296 & 371 & 359 & 404 & 212 \\
\hline $\mathrm{pH}$ & & 7.322 & 7.304 & 7.386 & 7.4 & 7.38 & 7.415 \\
\hline $\mathrm{pCO} 2(\mathrm{mmHg})$ & & 32.6 & 28.6 & 35.9 & 43 & 42 & 45.5 \\
\hline Bicarbonate $(\mathrm{mmol} / \mathrm{L})$ & & 16.5 & 13.9 & 21.1 & 26 & 24.3 & 28.5 \\
\hline Anion gap & & & & 19 & & & 10 \\
\hline Osmol (mOsm/kg) & & & & 317 & & & \\
\hline Serum ketones $(\mathrm{mmol} / \mathrm{L})$ & & & 6.1 & 4.3 & 0.7 & & \\
\hline White blood cell (/uL) & & 15,070 & 20,110 & & 7660 & 4880 & 2900 \\
\hline Hemoglobin (g/dL) & & 13.8 & 12.5 & & 34.9 & 35.3 & 34.7 \\
\hline Platelet (/uL) & & 182,000 & 201,000 & & 134,000 & 119,000 & 135,000 \\
\hline Procalcitonin (ng/mL) & & 3.4 & & & 13.27 & 19.5 & 37.04 \\
\hline C-reactive protein $(\mathrm{mg} / \mathrm{L})$ & & 193.02 & & & & & 291.41 \\
\hline Lactate $(\mathrm{mmol} / \mathrm{L})$ & & & 1.7 & 1.4 & & & \\
\hline BUN (mg/dL) & 26.3 & 27.7 & 30.8 & 34.5 & 34.9 & 35.3 & 34.7 \\
\hline Creatinine (mg/dL) & 2.46 & 3.99 & 5.13 & 4.92 & 4.65 & 3.33 & 1.91 \\
\hline eGFR $\left(\mathrm{mL} / \mathrm{min} / 1.73^{2}\right)$ & 27.66 & 15.83 & 11.83 & 12.42 & 13.27 & 19.5 & 37.04 \\
\hline
\end{tabular}


postoperative day 17 . His renal function recovered gradually and no sequelae were noticed on 1-year follow-up.

High anion gap metabolic acidosis and ketonemia are the diagnostic adjuncts of DKA. While significant hyperglycemia $(\geq 250 \mathrm{mg} / \mathrm{dL}$ ) due to insulin deficiency remains the core etiology for this disease, mild hyperglycemia or even a euglycemic status could not completely exclude its clinical existence. DKA without marked hyperglycemia was defined as euglycemic DKA, which may manifest with subtle symptoms from dehydration and is usually induced by hyperglycemic status $[1,3]$.

Currently, one of the known risk factors for euglycemic DKA is the use of SGLT2 inhibitors. While the pathogenesis still remains unclear, excess urinary glucose excretion with relatively lower plasma glucose concentration may lead to an imbalance between insulin and glucagon (less insulin production from the beta cells, and further stimulation of the alpha cells). Moreover, the SGLT2 inhibitors can act independently on alpha cells with higher glucagon concentrations and eventually contribute to hepatic ketogenesis [3-8].

In an extensive review, most patients presenting with DKA after bariatric surgery were reported to have type 1 diabetes or were on treatment with SGLT2 inhibitors. This condition was extremely rare to be associated with T2DM morbidly obese patients or in the absence of SGLT2 inhibitor treatment [1]. General anesthesia, associated surgical stress, abrupt discontinuation of insulin, postoperative infection, prolonged starvation, poor oral intake, or severe dehydration may predispose DKA for those undergoing bariatric surgery $[1,7]$.

Moreover, DKA has been reported to usually present 1 to 6 weeks after the initial bariatric surgery among T2DM patients $[2,7]$. Based on the similarity among manifestations of DKA and gastrointestinal symptoms due to bariatric surgeries, such as nausea or vomiting, timely identification and diagnosis may be difficult; nevertheless, our patient presented with euglycemic DKA sooner after initial RYGB (postoperative day 1), and the relative deficiency of insulin after this major surgery may be responsible for this condition. The recommendation of a basal insulin dosage of $0.3 \mathrm{U} / \mathrm{kg}$ of body weight during the perioperative stage as well as maintaining a target glucose level in the range of $140-180 \mathrm{mg} / \mathrm{dL}$ for the T2DM patients who will be undergoing bariatric surgery is essential [9]. In the early postoperative stage, diet modifications may be a key point for poor intake. Thus, dedicated titration of the insulin dose to avoid possible hypoglycemia is equally important [10].

Infection is another common predisposing factor for DKA, and procalcitonin can be a discriminatory biomarker for the detection of possible bacterial infection with concomitant leukocytosis and neutrophilia, which usually presents among those with DKA [2]. However, a previous case of a patient with DKA that had moderate elevation of procalcitonin without existing bacterial infection has been reported [11]. Furthermore, an acute attack of chronic gouty arthritis with febrile condition in our patient with concomitant euglycemic DKA could have been responsible for the relatively high procalcitonin level $(37.04 \mathrm{ng} / \mathrm{mL})[12,13]$.

The rare occurrence of euglycemia DKA and complex manifestations of concurrent chronic kidney disease with acute exacerbation and a gout attack confused us during the first 3 days after initial RYGB. Though extremely rare, clinicians should not neglect the possibility of DKA for morbidly obese patients with T2DM after bariatric surgery, even with a euglycemic status and without a history of SGLT2 inhibitor treatment.

Acknowledgements The authors would like to thank the enago (www. enago.tw) for their assistance with the language and grammar editing work.

\section{Declarations}

Statement of Informed Consent Informed consent was obtained from all individual participants included in the study.

Statement of Human and Animal Rights All procedures performed in studies involving human participants were in accordance with the ethical standards of the institutional and/or national research committee and with the 1964 Helsinki Declaration and its later amendments or comparable ethical standards.

Conflict of Interest The authors declare no competing interests.

Open Access This article is licensed under a Creative Commons Attribution 4.0 International License, which permits use, sharing, adaptation, distribution and reproduction in any medium or format, as long as you give appropriate credit to the original author(s) and the source, provide a link to the Creative Commons licence, and indicate if changes were made. The images or other third party material in this article are included in the article's Creative Commons licence, unless indicated otherwise in a credit line to the material. If material is not included in the article's Creative Commons licence and your intended use is not permitted by statutory regulation or exceeds the permitted use, you will need to obtain permission directly from the copyright holder. To view a copy of this licence, visit http://creativecommons.org/licenses/by/4.0/.

\section{References}

1. Aminian A, Kashyap SR, Burguera B, et al. Incidence and clinical features of diabetic ketoacidosis after bariatric and metabolic surgery. Diabetes Care. 2016;39(4):e50-3.

2. Rizo IM, Apovian CM. Diabetic ketoacidosis post bariatric surgery. Front Endocrinol (Lausanne). 2018;9:812.

3. Plewa MC, Bryant M, King-Thiele R. Euglycemic diabetic ketoacidosis. 2020 Apr 30. In: StatPearls. Treasure Island: StatPearls Publishing; 2020.

4. Peters AL, Buschur EO, Buse JB, et al. Euglycemic diabetic ketoacidosis: a potential complication of treatment with sodium- 
glucose cotransporter 2 inhibition. Diabetes Care. 2015;38(9): 1687-93.

5. Rosenstock J, Ferrannini E. Euglycemic diabetic ketoacidosis: a predictable, detectable, and preventable safety concern with SGLT2 inhibitors. Diabetes Care. 2015;38(9):1638-42.

6. Burke KR, Schumacher CA, Harpe SE. SGLT2 inhibitors: a systematic review of diabetic ketoacidosis and related risk factors in the primary literature. Pharmacotherapy. 2017;37(2):187-94.

7. Thiruvenkatarajan V, Meyer EJ, Nanjappa N, et al. Perioperative diabetic ketoacidosis associated with sodium-glucose cotransporter-2 inhibitors: a systematic review. Br J Anaesth. 2019;123(1):27-36.

8. Hoenes C, Rashid Q, Pimentel J. Diabetic ketoacidosis in a postoperative gastric bypass patient. J Surg Case Rep. 2017;2017(7): rjx148.

9. Busetto L, Dicker D, Azran C, et al. Practical recommendations of the obesity management task force of the European Association for the study of obesity for the post-bariatric surgery medical management. Obes Facts. 2017;10(6):597-632.

10. Mulla CM, Baloch HM, Hafida S. Management of diabetes in patients undergoing bariatric surgery. Curr Diab Rep. 2019;19(11): 112.

11. Cipriano A, Rebelos E, Park N, et al. Moderate increase of serum levels of procalcitonin in diabetic ketoacidosis. Neth J Med. 2018;76(10):454.

12. Zhang J, Zhao C, Wu T, et al. Procalcitonin may not be a differential diagnostic marker for bacterial infection in febrile patients with chronic gouty arthritis. J Int Med Res. 2018;46(10):4197-206.

13. Liu W, Sigdel KR, Wang Y, et al. High level serum procalcitonin associated gouty arthritis susceptibility: from a Southern Chinese Han population. PLoS One. 2015;10(7):e0132855.

Publisher's Note Springer Nature remains neutral with regard to jurisdictional claims in published maps and institutional affiliations. 